\title{
Escola Inter-relacional de Fibromialgia: Aprendendo a Lidar com a Dor - Estudo Clínico Randomizado
}

\section{Interactional School of Fibromyalgia: Learning to Cope with Pain - a Randomized Controlled Study}

\author{
Juliana Barcellos de Souza ${ }^{(1)}$, Patricia Bourgault( ${ }^{(2)}$, Jacques Charest ${ }^{(3)}$, Serge Marchand ${ }^{(4)}$
}

\begin{abstract}
RESUMO
Objetivo: Avaliar os efeitos tardios de um programa interdisciplinar para pacientes com síndrome de fibromialgia (FM) e comparar esses efeitos com o tratamento tradicional (acompanhamento médico, exames, medicamentos). Método: $\mathrm{O}$ tratamento proposto, denominado Escola Inter-relacional de Fibromialgia (EIF), consiste de um programa interdisciplinar de 11 semanas, com nove encontros com grupos de oito pacientes e dois profissionais da saúde. Trinta mulheres com FM participaram do grupo experimental e outras 30 do grupo-controle. Ambos os grupos foram avaliados em três momentos distintos: antes da EIF, imediatamente após e quatro meses após a alta. As pacientes que constituíam o grupo experimental foram também reavaliadas 12 meses após obterem alta. As variáveis dependentes foram: dor clínica e nível de disposição (ambas adquiridas por escalas visuais analógicas), cinco escalas do inventário multidimensional da dor (gravidade da dor, interferência da dor nas atividades cotidianas, percepção de controle da vida, distúrbios emocionais e suporte familiar/social). Resultados: Com taxa de participação de $97 \%$, as pacientes do grupo experimental apresentaram melhora significativa após o tratamento e nas avaliações seguintes. Esta melhora significativa do quadro clínico também foi constatada quando comparada à evolução das pacientes do grupocontrole. Por meio da análise das variâncias $(2 \times 3)$ nos dados da amostra tratada, e teste $t$ de Student pareado para a população intent-to-treat, percebe-se que o grupo experimental apresenta redução significativa das dores (todos os $\mathrm{p}<0,05$ ). A melhora das pacientes durante o tratamento manteve-se inalterada até um ano após a alta. Conclusão: O programa da EIF permite a obtenção de melhora significativa e estável na condição clínica das pacientes com FM. Com o artigo, segue discussão sobre estratégias para favorecer a participação ativa das pacientes.
\end{abstract}

Palavras-chaves: fibromialgia, tratamento multidisciplinar em grupo, efeito a longo prazo, participação ativa, adesão ao tratamento.

\begin{abstract}
Objective: To assess long-term efficacy of an interdisciplinary group intervention of patients with fibromyalgia (FM) as compared with (Canadian) standard medical care. Methods: The program, called Interactional School of Fibromyalgia (ISF), consists of 9 meetings (during 11 weeks) with eight participants and two facilitators. Sixty women with FM were randomly assigned to one of two groups (ISF or Control group). Outcomes variables were: clincal pain and energy (Visual analogue scale and five Multidimentional Pain Iventory (Pain severtity, Pain iterference, Life control, Affective distress and Support from significant others). These variables were measured at baseline; immediately after the program; 4 months and 1 year later. Results: Efficacy analyses from completers showed a treatment effect on measures even after one year. The aderence of ISF was 97\%. Analyses of variance $(2 \times 3)$ and Intent-to-treat analyses indicate that ISF group experienced lower pain (all ps <0,05) even at the one year follow up. Conclusion: These findings demonstrate the long-term efficacy of this group intervention. Strategies to increase patients' adherence were discuted.
\end{abstract}

Keywords: fibromyalgia, interdisciplinary group intervention long term effects, active participation of patients, treatment adherence.

Recebido em 12/1 1/2007. Aprovado, após revisão, em 1/6/2008. Conflitos de interesse: Agências financiadoras: Coordenação de Aperfeiçoamento de Pessoal de Nível Superior (Capes): bolsa de doutorado pleno no exterior para JBS; Canadian Institutes of Health Research (CIHR).

1. Fisioterapeuta, doutorado, Université de Sherbrooke.

2. Enfermeira, doutorado pela Université du Québec en Abitibi-Témiscamingue

3. Psicólogo, doutorado, Université du Québec en Abitibi-Témiscamingue.

4. Neurofisiologista, doutorado, Université de Sherbrooke e Université du Québec en Abitibi-Témiscamingue.

Endereşo para correspondência: Serge Marchand, Équipe de Recherche sur la douleur - CRC-CHUS, Université de Sherbrooke, Faculté de médecine et des Sciences de la Santé, 3001, 12e avenue nord, Sherbrooke (Québec), Canada J1H 5N4. 


\section{INTRODUÇÃO}

A fibromialgia (FM) é uma síndrome de dor crônica que se manifesta em cerca de $2,5 \%$ dos brasileiros ${ }^{(1)}$ e $2 \%$ dos canadenses $^{(2)}$. De causa ainda desconhecida, o quadro clínico da FM agrega um conjunto de sintomas, como a presença de dor, fadiga, rigidez matinal, distúrbios do sono e do humor. Contudo, dois destes sintomas são marcantes para o diagnóstico, quais sejam, dor difusa persistente há mais de três meses consecutivos, associada à sensibilidade à palpação de pontos anatômicos precisos ( 11 dos 18 pontos devem ser dolorosos com a pressão equivalente a $4 \mathrm{~kg})^{(3)}$.

A complexidade da FM vai além do quadro clínico. Diversos estudos $^{(4,5)}$ propõem equipes multidisciplinares para tratar esta síndrome. Entre as características dos tratamentos já propostos pela literatura científica, destacam-se os programas com sessões de exercícios ${ }^{(6,7)}$, relaxamento $^{(8)}$, controle do estresse e terapia cognitiva comportamental ${ }^{(9,10)}$. Os programas interdisciplinares duram, em média, de um a seis meses e são geralmente realizados com grupos de pacientes, variando entre 10 e 25 pacientes por grupo. A escolha deste modelo de tratamento (multidisciplinar e em grupo) justifica-se pela melhora na qualidade de vida e na condição de saúde dos pacientes com FM ${ }^{(11,12)}$. No entanto, a proporção de pacientes que abandona os programas é significativa (um em cada três) ${ }^{(13,14)}$. Este nível de abandono dificulta a análise estatística dos resultados. Do ponto de vista clínico, a baixa adesão ao tratamento demonstra que os programas não estão suficientemente adaptados para atender as necessidades desta população, visto que os índices de abandono no contexto clínico são geralmente superiores àqueles encontrados nas pesquisas.

Além desses limites, os programas interdisciplinares agregam custo elevado em recursos humanos, exigindo a disponibilidade de vários profissionais da saúde, e em recursos de infra-estrutura, local e equipamentos adequados.

O grande desafio dos programas multidisciplinares consiste de ampliar a adesão ao tratamento, motivar os pacientes a manterem-se ativos, ou seja, favorecer a sua autonomia e minimizar, se possível, os custos agregados aos programas interdisciplinares, por exemplo, reduzir a duração do programa e o número de profissionais da saúde diretamente envolvidos.

Dessa forma, este estudo teve como objetivo identificar e avaliar os efeitos clínicos a curto e longo prazos de um programa multidisciplinar que atendesse as necessidades citadas anteriormente. Este programa, denominado Escola Inter-relacional de Fibromialgia (EIF), consiste de nove sessões e exige a presença de apenas dois profissionais da saúde para interagir com o grupo de oito pacientes.

\section{MATERIAIS E MÉTODOS}

Este estudo foi aprovado pelos comitês de ética em pesquisa com seres humanos das duas instituições onde se realizaram os experimentos. Todos os participantes foram voluntários e podiam se retirar do programa a qualquer momento, sem comprometer a continuidade do acompanhamento médico ou de qualquer outro serviço da saúde. Este estudo ocorreu em duas etapas, quais sejam, uma teórica, para a elaboração do programa interdisciplinar, e outra clínica, para avaliação dos seus efeitos clínicos a curto, médio e longo prazos. Avaliou-se a EIF por meio de um ensaio clínico randomizado com grupo-controle clinicamente equivalente e pela coleta de dados até um ano após a alta (Figura 1).

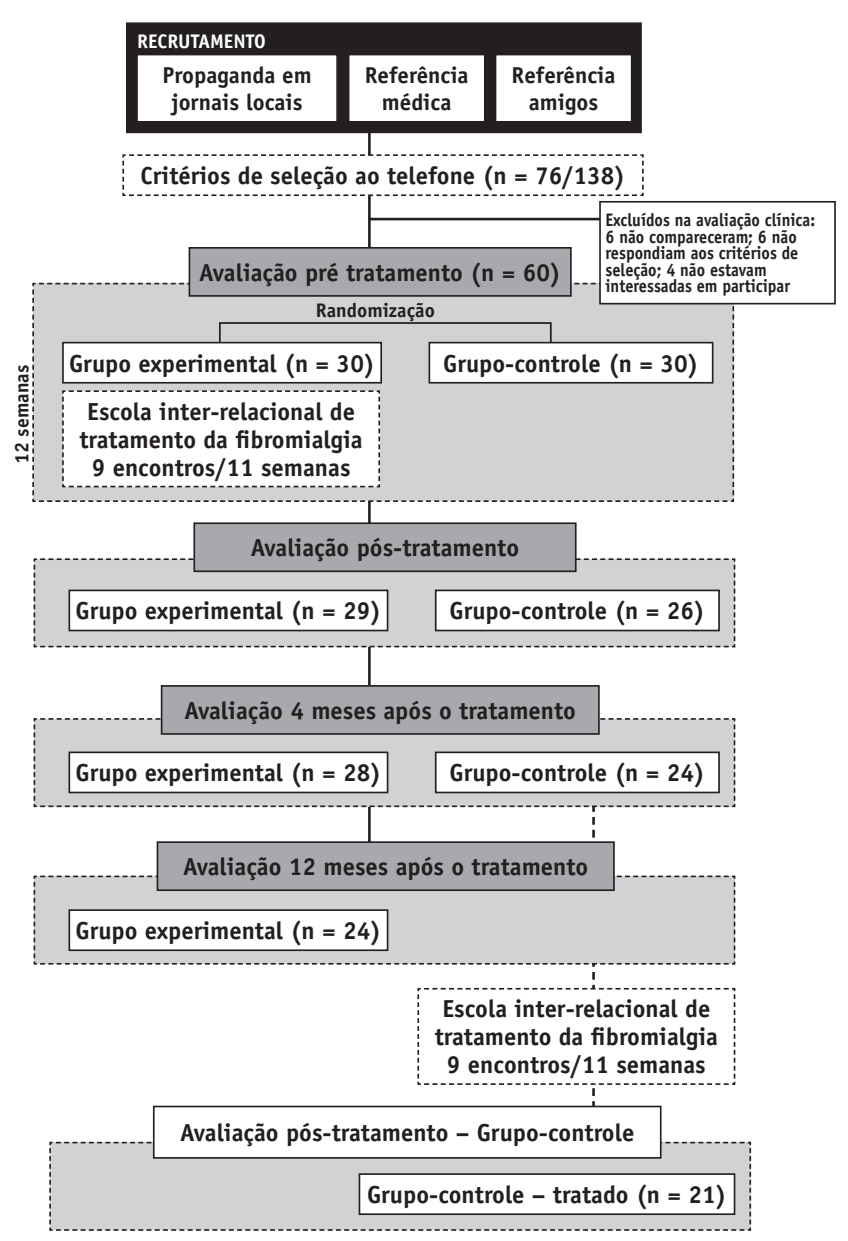

Figura 1 - Esquema representativo das etapas da pesquisa. 


\section{AMOSTRA}

Para a realização da EIF, foram recrutadas 60 mulheres com o diagnóstico de FM (> 6 meses), com idade entre 20 e 65 anos, que não tomavam medicamentos há mais de três meses ou com tratamento farmacológico estável $(>3$ meses). Esta estabilidade no consumo de medicamentos favorece o controle dos efeitos proporcionados pela EIF, evitando a inclusão de ruídos, em virtude de mudanças no tratamento farmacológico.

Pacientes com FM e comorbidades tipo depressão, lombalgia, hipotireoidismo, síndrome do estresse pós-traumático também foram incluídas no estudo. Sendo excluídas as mulheres gestantes e lactantes ou com comorbidades, como câncer, depressão grave associada a pensamentos suicidas (fase de planejamento), artrite reumática e cardiopatia não-controlada.

\section{VARIÁVEIS}

\section{INVENTÁRIO MULTIDIMENSIONAL DA DOR (IMD)}

Questionário auto-administrado que abrange os múltiplos aspectos psicossociais da dor crônica ${ }^{(15)}$. Cinco subescalas deste questionário foram utilizadas durante o presente estudo: gravidade da dor, interferência da dor nas atividades cotidianas, percepção de controle da vida, distúrbios emocionais (humor, irritabilidade) e suporte familiar/social. Os valores das subescalas variam de zero a seis. Quanto mais elevado o valor, maior o nível de importância agregado à variável. Em pacientes com dores crônicas os valores médios citados pela literatura científica são de $4,37$ ( $\pm 1,05), 4,71( \pm 0,93), 4,33( \pm 1,63), 3,12( \pm 1,32)$, e $3,55( \pm 1,26)$, respectivamente ${ }^{(16)}$.

\section{DOR CLÍNICA E DISPOSIÇÃO PARA}

REALIZAR ATIVIDADES COTIDIANAS

Estas variáveis foram mensuradas por meio de um instrumento denominado "diário da dor", preenchido pelo próprio paciente em seu domicílio ${ }^{(17,18)}$. O "diário da dor" consiste de três escalas visuais analógicas (EVA). Duas escalas fazem referência à dor: intensidade e aspecto psicológico (sofrimento associado à dor) e uma terceira que se refere à disposição para realizar atividades cotidianas. Cada escala é representada por uma linha horizontal de 100 milímetros com extremidades representando as condições "sem dor" (equivalente a zero) e "dor insuportável" ou "sofrimento associado à dor insuportável" (equivalente a $100 \mathrm{~mm}$ ) para as escalas de dor; ou ainda "sem disposição" e "extremamente disposta" para a percepção de disposição física e mental. As pacientes marcavam cada EVA no nível em que avaliavam a intensidade da dor (componente físico), o sofrimento a ela associado (componente psicológico) e a disposição física. Estas variáveis foram coletadas a cada duas horas, durante o período de três dias alternados de uma mesma semana. Cada variável foi calculada pela média dos valores obtidos durante os três dias.

\section{ANÁLISE DOS DADOS}

A priori, estabeleceu-se a necessidade de uma amostra de 24 pacientes em cada grupo para detectar diferença significativa de três pontos na EVA de intensidade da dor $(\alpha=$ $5 \%, \beta=10 \%)$. O tamanho da amostra foi calculado após o tratamento das 15 primeiras pacientes. Para a constituição dos dois grupos do estudo, as pacientes foram selecionadas aleatoriamente em um grupo experimental (que recebeu o tratamento da EIF) e o outro controle (em lista de espera). A análise estatística com o teste $t$ de Student para amostras independentes permitiu a validação desta randomização.

A eficácia da EIF a curto e médio prazos foi avaliada por uma série de análises de variância (ANOVA) (2 grupos $\times 3$ tempos de aquisição dos dados) na população $\mathrm{PP}$ ( per protocol). Análises intra e intersujeitos foram possíveis sempre que as ANOVAs $(2 \times 3)$ apresentavam-se significativa $(\mathrm{p}<0,05)$. Os efeitos da EIF a longo prazo foram analisados por meio de testes $t$ de Student para dados pareados com a população $\mathrm{PP}$ (pacientes que completaram o estudo, $\mathrm{n}=24)$ e com a população constituída pelo princípio do intent to treat (ITT). A população do ITT foi composta pelas pacientes que concluíram o estudo, aquelas que abandonaram o tratamento e as que não retornaram ao laboratório para as avaliações após o tratamento $(\mathrm{n}=30)$. Todos os dados foram analisados com o SPSS for Windows versão 14 , aceitando $5 \%$ como nível de significância.

\section{PROGRAMA ESCOLA INTER-RELACIONAL}

DE TRATAMENTO DA FIBROMIALGIA

A EIF consiste de um programa interdisciplinar para tratar grupos de pacientes com FM, tendo como meta ensinar técnicas de autotratamento aos pacientes. O programa tem duração de 11 semanas, período em que ocorrem nove sessões em grupo (descrição detalhada na Tabela 1).

Ao contrário dos tratamentos propostos anteriormente, fundamentados na terapia cognitiva comportamental, o programa em questão fundamenta-se no modelo interrelacional proposto pelas teorias da comunicação ${ }^{(19) \cdot}$ Neste modelo, o indivíduo/paciente em interação com o seu 
TABELA 1

DESCRIÇÃO DO PROGRAMA: ESCOLA INTER-RELACIONAL DE FIBROMIALGIA (EIF)

\begin{tabular}{|c|c|}
\hline Encontro & Assunto de discussão \\
\hline 1. Contrato terapêutico & $\begin{array}{l}\text { Apresentar brevemente as nove etapas do programa e negociar o contrato terapêutico. Este contrato tem como objetivo } \\
\text { modular as expectativas dos pacientes e consiste de: a) definir três objetivos pessoais realistas e mensuráveis; b) determi- } \\
\text { nar qual a menor porcentagem de melhora, no quadro clínico, aceitável (entre } 5 \% \text { e } 20 \% \text { ). Com o contrato, os participan- } \\
\text { tes também se comprometem a dedicar } 45 \text { minutos/dia, } 6 \text { dias/semana às atividades prescritas pela EIF. } \\
\text { Praticar em grupo a respiração diafragmática. Esta deve ser praticada diariamente ( } 3 \text { vezes ao dia, durante } 5 \text { minutos) }{ }^{*} \text {. }\end{array}$ \\
\hline 2. Sintomas & $\begin{array}{l}\text { Discutir os principais sintomas; descrição do ciclo de "dor-fadiga-estresse-insônia-dor"; discussão em grupo sobre as estra- } \\
\text { tégias (não-farmacológicas) utilizadas pelos participantes, para o tratamento dos seus sintomas. } \\
\text { Durante as semanas que seguem, os pacientes devem praticar duas novas estratégias propostas pelo grupo e continuar com } \\
\text { os treinos de respiração*. }\end{array}$ \\
\hline 3. Preparação física & $\begin{array}{l}\text { Prescrever um programa de exercício a ser realizado em domicílio*: a) rotina de exercício } 6 \text { vezes/semana, } 15 \text { minutos, } \\
\text { com alongamento e fortalecimento; b) caminhada, intensidade moderada } 40 \% \text { a } 60 \% \mathrm{FC}_{\operatorname{máx}}{ }^{\prime} 3 \text { vezes/semana; } 30 \text { minutos. } \\
\text { Estes exercícios também devem ser realizados semanalmente. }\end{array}$ \\
\hline 4. Preparação mental & $\begin{array}{l}\text { Escolher uma técnica de relaxamento e praticá-la } 3 \text { vezes/semana durante } 15 \text { a } 20 \text { minutos. A EIF apresenta alguns méto- } \\
\text { dos de relaxamento*. }\end{array}$ \\
\hline 5. Respeitar seus limites & $\begin{array}{l}\text { Discutir e prescrever estratégias para respeitar seu nível de energia/disposição, ou seja, recusar, aceitar ou delegar uma } \\
\text { tarefa, assim como observar-se e valorizar-se*. }\end{array}$ \\
\hline 6. Nutrição & $\begin{array}{l}\text { Propor dieta saudável e equilibrada, com ênfase no consumo de proteína a cada refeição }(15 \mathrm{~g} / \text { refeição como sugere o } \\
\text { Guia Canadense de Nutrição) }{ }^{\star} \text {. }\end{array}$ \\
\hline \multicolumn{2}{|c|}{2 semanas de trabalho autônomo: integração das estratégias de maneira individual (sem encontro com os terapeutas). } \\
\hline 7. Consequências da cronicidade & Discutir sobre sexualidade, depressão e pensamentos suicidas. \\
\hline 8. Tratamento & Discutir sobre os efeitos imediatos e tardios dos tratamentos farmacológicos e dos não-farmacológicos \\
\hline 9. Retrospectiva & $\begin{array}{l}\text { Motivar os pacientes a continuar as atividades, por meio da retrospectiva à condição inicial. Os pacientes devem verbalizar } \\
\text { "o que pode ser feito para que eu volte a sentir dores como antes do programa da EIF?" Dessa forma, verbalizam o que não } \\
\text { deve ser feito: abandonar as atividades. }\end{array}$ \\
\hline
\end{tabular}

* Essas atividades são prescritas de maneira cumulativa e devem ser mantidas obrigatoriamente durante todo o período de 11 semanas da EIF (9 encontros + 2 semanas de pausa).

sistema pode permanecer temporariamente autônomo, independentemente da sua condição inicial. Parte-se do princípio de que o tratamento deve se concentrar na situação presente (comportamento atual) em vez de fixar-se à condição inicial (passado). O ponto crucial desta terapia é a persistência do problema (no caso, a dor crônica e sua influência psicossocial) e não a sua origem (ou seja, a causa ou o evento que desencadeou a FM) ${ }^{(20)}$.

Outro teorema da teoria de comunicação utilizado durante a EIF é a interação entre os elementos do sistema que - de tão estreitas mudanças no comportamento do paciente - implicará modificações no comportamento dos outros (por exemplo: mudanças na dinâmica entre os membros da família do paciente).

Além destes teoremas, a EIF parte do princípio que é "impossível não se comunicar". Geralmente, acredita-se que a comunicação resume-se à troca de mensagens verbais. No entanto, a maior parte da comunicação ocorre por meio de mensagens não-verbais ${ }^{(21)}$. Os terapeutas devem ser vigilantes aos efeitos que produzem sobre os pacientes, pelo simples fato de estar em relação terapêutica. Este modelo de tratamento é centralizado na interação, na qual diversas estratégias terapêuticas podem ser utilizadas para influen- ciar positivamente o paciente. Atualmente, desconhece-se outro programa que tenha utilizado estas noções para o tratamento da FM. Contudo, este modelo inter-relacional já foi utilizado, associado a um programa multidisciplinar, para a lombalgia crônica. Os resultados demonstraram eficácia na manutenção dos efeitos do tratamento a médio prazo (seis meses) e com excelente adesão ao tratamento $(12 \% \text { de abandono })^{(18,22)}$.

O programa da EIF tem duração de 11 semanas, período em que ocorrem as nove etapas do tratamento. Cada sessão tem duração de duas horas e ocorrem semanalmente. Entretanto, há uma pausa de 15 dias entre a sexta e a sétima sessões. Esta pausa no tratamento favorece a integração das atividades prescritas e desenvolvidas durante a EIF no cotidiano de cada indivíduo. As sessões ocorrem com grupos de sete a oito pacientes, com a supervisão e a orientação de dois profissionais da saúde (por exemplo: fisioterapeuta e enfermeiro ou fisioterapeuta e psicólogo). Ao contrário de estudos anteriores, durante a EIF é importante que os pacientes sigam o tratamento sempre com o mesmo grupo, ampliando, assim, a interação entre eles e a influência de uns sobre os outros. 


\section{RESULTADOS}

A amostra foi composta por 60 mulheres de 49,6 67 anos de idade, com queixas de dores crônicas há 12,4 \pm 9 anos e com diagnóstico de FM há 6,6 \pm 5 anos. Dezessete pacientes não faziam nenhum tipo de tratamento farmacológico, ou seja, não recorriam a medicamentos para alívio dos sintomas da FM. Quarenta pacientes justificaram o aparecimento da FM como conseqüência de trauma de origem psicológica, física ou ambos, enquanto as outras 20 pacientes dissociaram a síndrome de qualquer evento ou trauma vivido. Vinte e oito pacientes trabalhavam (tempo integral ou parcial). Na avaliação inicial, a maioria das pacientes $(n=40)$ considerava a atividade física um método eficaz para o controle dos sintomas, porém apenas nove faziam atividade física regularmente (de duas a três vezes por semana). O método de randomização para a constituição dos grupos (controle e experimental) demonstrou-se eficaz, pois os valores de todas as variáveis adquiridas na avaliação inicial eram equivalentes (todos os $p>0,17$ ).
Durante a fase experimental, todas as pacientes do grupo experimental eram fisicamente ativas, e realizaram semanalmente as atividades propostas pelo programa. Paralelamente, as pacientes do grupo-controle mantiveram sua rotina em busca de tratamentos tradicionais e/ou alternativos para a FM, ou seja, 37\% mudaram seu tratamento farmacológico, $37 \%$ iniciaram a prática de caminhadas (sem orientação profissional), 7\% receberam infiltrações de cortisona e 7\% tomaram a iniciativa de praticar meditação ou outra técnica de relaxamento mental.

No grupo experimental, observaram-se efeitos benéficos significativos em três das cinco escalas do IMD, ou seja, gravidade da dor, interferência da dor nas atividades de vida diária e percepção de controle da vida (todos os $\mathrm{p}<0,05$ ) (Figura 2). Nenhuma modificação foi observada nas variáveis dos distúrbios emocionais $(\mathrm{F}=1,834 ; \mathrm{p}=0,17)$ nem no suporte familiar/social $(\mathrm{F}=0,044 ; \mathrm{p}=0,957)$.

Por meio da análise dos dados com a população ITT, percebeu-se que os efeitos clínicos a longo prazo proporcio-
A

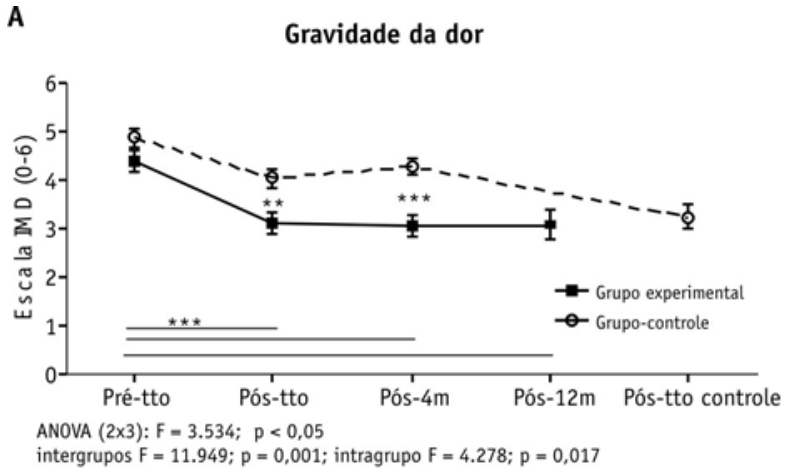

C

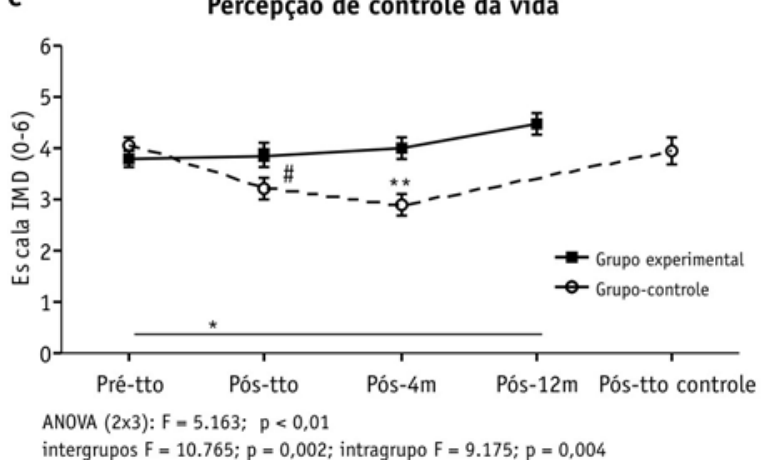

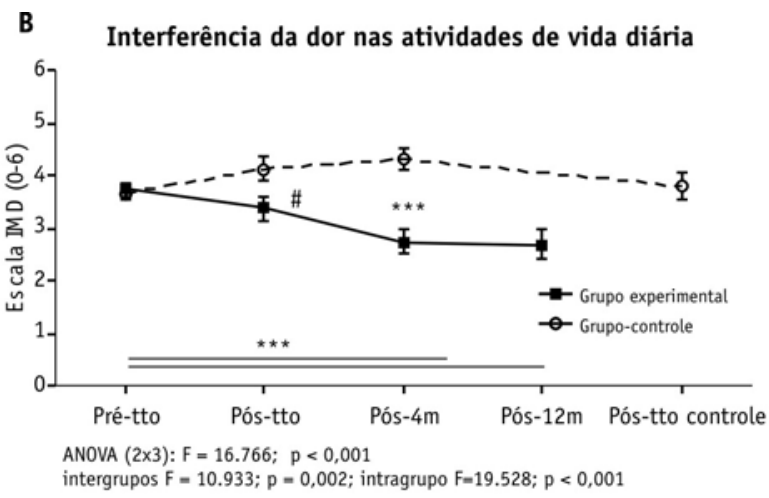

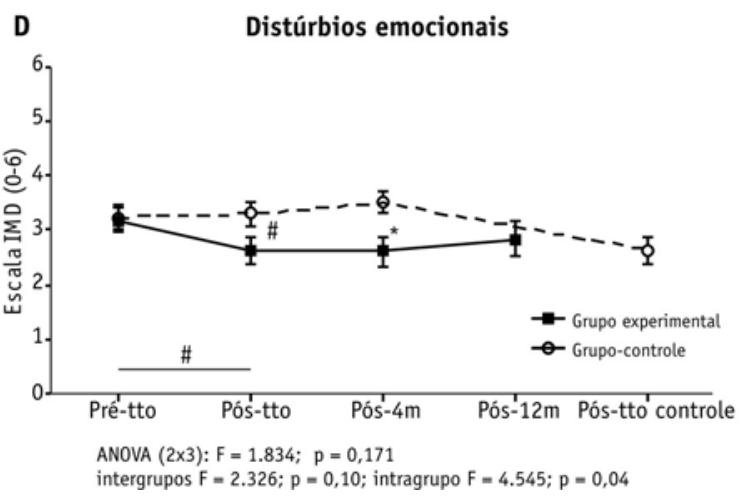

$\# p<0,05 ;{ }^{*} p<0,01 ;{ }^{* *} p<0,001 ; * * * p<0,0001$.

Figura 2 - Gráficos ilustrando evolução de quatro subescalas do inventário multidimensional da dor: A) gravidade da dor; B) interferência da dor nas atividades diárias; C) percepção de controle da sua vida e (D) distúrbios emocionais. 
nados pela EIF mantiveram-se significativos para algumas escalas do IMD, como a gravidade da dor e a interferência da dor nas atividades de vida diária (ambos $\mathrm{p}<0,01$ ). Porém, a percepção de controle da vida, os distúrbios emocionais e o suporte familiar/social não apresentaram efeitos significativos após um ano da alta $(\mathrm{p}=0,15$, $\mathrm{p}=0,30$ e $\mathrm{p}=0,54$, respectivamente).

A dor clínica reduziu-se, de modo significativo, após o tratamento e manteve-se baixa ao longo dos 12 meses após a alta. Este padrão de melhora pôde ser observado tanto na intensidade quanto no aspecto psicológico da dor $(\mathrm{p}<0,05)$. Da mesma forma, a disposição na realização das tarefas diárias aumentou significativamente após o tratamento (Figura 3). Estes resultados da população tratada (PP) são equivalentes aos da população ITT (todos os $\mathrm{p}<0,01$ ).

\section{DISCUSSÃO}

O programa multidisciplinar da EIF demonstrou efeitos clínicos estatisticamente significantes, para a redução na percepção da dor e para o aumento da disposição física e mental dos pacientes. A melhora observada nos distúrbios de humor e na percepção de controle dos sintomas é notável um ano após a alta. Todas as pacientes que concluíram o tratamento (29 de 30 ) apresentaram melhoras na sua condição clínica. Cabe ressaltar que apenas uma paciente abandonou o tratamento, não retornando aos encontros após a pausa de duas semanas, o que representa índice de abandono de 3\%, índice inferior àqueles observados em estudos anteriores.

Este índice demonstra o quanto o programa proposto é versátil. Apesar de o modelo de tratamento ser calçado na interação - utilizando estratégias terapêuticas para influenciar os pacientes -, percebe-se que este tratamento pode atender às necessidades básicas da população com FM.

O sucesso terapêutico depende, invariavelmente, da colaboração e da participação ativa dos pacientes na realização das tarefas prescritas, sejam elas atividades físicas, de respiração, relaxamento ou atividades cognitivas. A importância da taxa de desistência dos tratamentos multidisciplinares motiva alguns pesquisadores a excluir, de suas análises estatísticas, aqueles pacientes cuja assiduidade seja inferior a $45 \%^{(9,23)}$. Esta exclusão dos participantes visa a evitar subestimação dos resultados obtidos.

Ao contrário de estudos precedentes, optou-se por incluir (na análise estatística) os dados de todos as pacientes que se apresentaram nas avaliações no laboratório, independentemente de seu nível de atividade. Além disso, para controlar resultados sub ou supra-estimados, realizou-se testes estatísticos com a população inicial (ITT) para verificar os efeitos clínicos tardios da EIF. Qualitativamente, as pacientes tendem a adaptar as atividades prescritas às suas necessidades. Por exemplo, algumas pacientes aumentaram a freqüência da caminhada para seis vezes por semana (em vez de três vezes), enquanto outras optaram pela meditação diária. Estas alterações

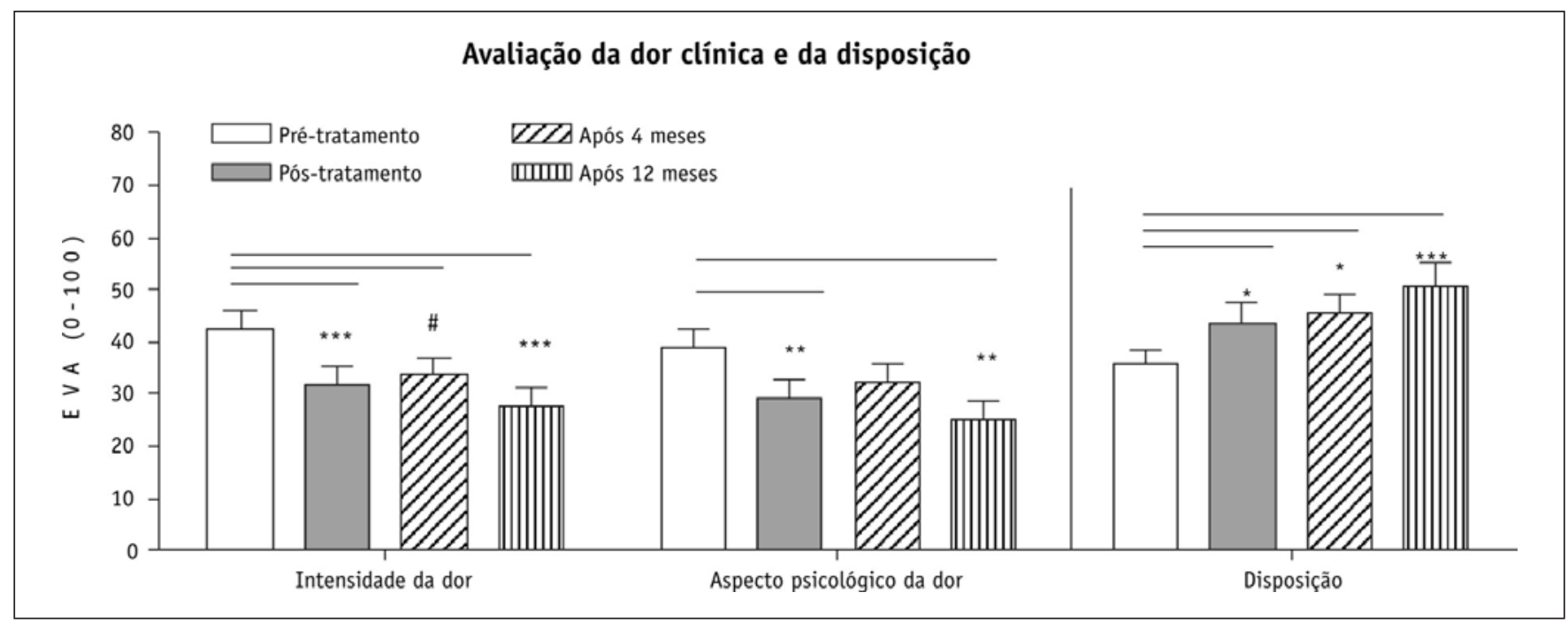

$\# p<0,05 ;{ }^{*} p<0,01 ;{ }^{* *} p<0,001 ;{ }^{* * *} p<0,0001$.

Figura 3 - Gráfico ilustrando a variação da intensidade da dor, do aspecto psicológico da dor e da disposição para realizar atividades, relatadas pelas pacientes do grupo experimental. 
no programa inicial da EIF demonstram a autonomia da paciente diante de seu tratamento. Os benefícios dessas modificações podem ser confirmados pelos resultados coletados durante este estudo.

Para estimular a adesão e a participação ativa das pacientes ao tratamento foram propostas cinco estratégias ao longo do programa da EIF. Primeiramente, acreditar na dor da paciente e confirmar que sua dor seja real. Estas pacientes sofrem de síndrome de dor crônica incapacitante e invisível, convivem com a incerteza do diagnóstico e com a suposição de viver com uma "dor imaginária"(24).

Outra estratégia foi estabelecer, com a paciente, objetivos realistas e mensuráveis, plausíveis de serem realizados até o final do tratamento. A motivação do paciente pode ser ampliada, quando se estabelecem metas específicas. Por exemplo, se o paciente deseja "fazer longas caminhadas em terrenos irregulares - trilhas em contato com natureza", o terapeuta, por sua vez, deve auxiliá-lo a reformular este objetivo, para "fazer caminhada de uma hora e trinta minutos em terreno irregular sem morros, uma vez por mês". O objetivo deve ser viável e adaptar-se à condição física do paciente, devendo ser validado ao final do tratamento. Atingir ao menos um dos objetivos pessoais estabelecidos é outro elemento crucial, para que o paciente perceba a sua eficiência no autotratamento e persista com as atividades da EIF, mesmo após a alta.

A terceira estratégia consistiu de elaborar o plano de ação ou plano de tratamento, que tem como base um planejamento simples - descrevendo a atividade, sua freqüência

\section{REFERÊNCIAS}

1. Senna ER, De Barros AL, Silva EO, et al.: Prevalence of rheumatic diseases in Brazil: a study using the COPCORD approach. J Rheumatol 31: 594-7, 2004.

2. McNally JD, Matheson DA, Bakowsky VS: The epidemiology of self-reported fibromyalgia in Canada. Chronic Dis Can 27: 9-16, 2006.

3. Wolfe F, Smythe HA, Yunus, MB, et al.: The American College of Rheumatology 1990 criteria for the classification of fibromyalgia. Report of the multicenter criteria committee. Arthritis Rheum 33: 160-72, 1990.

4. Henriksson CM: Living with continuous muscular pain-patient perspectives. Part II: strategies for daily life. Scand J Caring Sci 9: 77-86, 1995.

5. Turk DC, Dworkin RH, Allem RR, et al.: Core outcome domains for chronic pain clinical trials: IMMPACT recommendations. Pain 106: 337-45, 2003.

6. Da Costa D, Abrahamowicz M, Lowensteyn I, et al.: A randomized clinical trial of an individualized home-based exercise e como incluí-la em sua rotina -, que ajuda diretamente o paciente a melhorar sua condição crônica ${ }^{(25,26)}$.

A quarta estratégia foi construir e manter uma aliança terapêutica afetiva, ou seja, ser flexível nas prescrições e favorecer a relação complementar (não-hierárquica) ${ }^{(27)}$.

Finalmente, a quinta estratégia foi prescrever atividades simples e de efeito rápido. Por exemplo, respirar lentamente para relaxar, ou combater a fadiga ingerindo maior quantidade de proteínas, evitando, assim, os picos de energia proporcionados pela ingestão de açúcares e carboidratos.

Embora este estudo tenha sido realizado com grupos de pacientes, estas estratégias - que visam a ampliar a adesão dos pacientes ao tratamento - também podem ser utilizadas em tratamentos individuais.

Estudos futuros devem ampliar a população para mensurar os efeitos tardios, incluindo homens com FM na amostra, e, além disso, testar este modelo de terapia, para atendimento individualizado em consultório.

\section{AGRADECIMENTOS}

Os autores agradecem as agências financiadoras Coordenação de Aperfeiçoamento de Pessoal de Nível Superior (Capes) - bolsa de doutorado pleno no exterior para JBS - e Canadian Institutes of Health Research (CIHR) e Fonds de Recherche en Santé Québec (FRSQ) - fundos de pesquisa de SM; e os membros da equipe Michelle Provencher, Édith Normand, Paule Julien, Stéphanie Pagé, Marie-Claude Tremblay, Serge Daigle, Nancy Julien, Sylvie L'Heureux, Christine Brown e Gertrudes Maria de Oliveira Cardoso e Silva.

programme for women with fibromyalgia. Rheumatology (Oxford) 44: 1422-7, 2005.

7. Dobkin PL, Abrahamowicz M, Fitzcharles MA, et al.: Maintenance of exercise in women with fibromyalgia. Arthritis Rheum 53: 724-31, 2005.

8. Keel PJ, Bodoky C, Gerhard U, Muller W: Comparison of integrated group therapy and group relaxation training for fibromyalgia. Clin J Pain 14: 232-8, 1998.

9. Gowans SE, Dehueck A, Voss S, et al.: Effect of a randomized, controlled trial of exercise on mood and physical function in individuals with fibromyalgia. Arthritis Rheum 45: 519-29, 2001 .

10. Bennett RM, Burckhardt CS, Clark SR, et al.: Group treatment of fibromyalgia: a 6 month outpatient program. J Rheumatol 23: $521-8,1996$.

11. Mannerkorpi K, Nuberg B, Ahlmen M, Ekdahl C: Pool exercise combined with an education program for patients with fibromyalgia syndrome. A prospective, randomized study. J Rheumatol 27: 2473-81, 2000. 
12. Lemstra M, Olszynski WP: The effectiveness of multidisciplinary rehabilitation in the treatment of fibromyalgia: a randomized controlled trial. Clin J Pain 21: 166-74, 2005.

13. Dobkin PL, Da Costa D, Abrahamowicz M, et al.: Adherence during an individualized home based 12 -week exercise program in women with fibromyalgia. J Rheumatol 33: 333-41, 2006.

14. Wigers SH, Stiles TC, Vogel PA: Effects of aerobic exercise versus stress management treatment in fibromyalgia. A 4.5 year prospective study. Scand. J Rheumatol 25: 77-86, 1996.

15. Kerns RD, Turk DC, Rudy TE: The West Haven-Yale Multidimensional Pain Inventory (WHYMPI). Pain 23: 345-56, 1985.

16. Laliberté S, Sullivan MJL, Charron J, et al.: Multidimensional pain inventory à l'inventaire multidimensionnel de la douleur: traduction et validation. Canadian Pain Society Meeting. 2005. Ref type: abstract.

17. Marchand S, Charest J, Li J, Chenard JR, et al.: Is TENS purely a placebo effect? A controlled study on chronic low back pain. Pain 54: 99-106, 1993.

18. Charest J, Lavignolle B, Chenard J-R, et al.: École interactionnelle du dos. Rhumatologie 48: 221-37, 1994.

19. Bertalanffy LV: General system theory - a critical review. In: Bertalanffy LV, Rapoport A, editores. General systems. New York: Society for General Systems Research; 1962. p. 1-20.
20. Weakland JH: Thérapie systémique individuelle. In: Watzlawick P., Nardone, G., editores. Stratégie de la thérapie brève Paris, Seuil; 2000. p. 155-69.

21. Fisch R, Schlanger K: Traiter les cas difficiles. Paris: Seuil; 2005.

22. Charest J, Chenard J-R, Lavignolle B, Marchand S: Lombalgie: école interactionnelle du dos. Paris: Masson; 1996.

23. Jones KD, Burckhardt CS, Clark SR, et al.: A randomized controlled trial of muscle strengthening versus flexibility training in fibromyalgia. J Rheumatol 29: 1041-8, 2002.

24. Sturge-Jacobs M: The experience of living with fibromyalgia: confronting an invisible disability. Res Theory Nurs Pract 16: 19-31, 2002.

25. Handley M, Macgrefor K, Schillinger D, et al.: Using action plans to help primary care patients adopt healthy behaviors: a descriptive study. J Am Board Fam Med 19: 224-31, 2006.

26. Steihaug S: Women's strategies for handling chronic muscle pain: A qualitative study. Scand J Prim Health Care 25: 44-8, 2007.

27. Baillargeon P, Pinsof W-M, Leduc A: Modèle systémique de l'alliance thérapeutique. Revue Européenne de Psychologie Appliquée 55: 137-43, 2005. 